\title{
Noradrenaline Unmasks Novel Self-Reinforcing Motor Circuits within the Mammalian Spinal Cord
}

\author{
David W. Machacek and Shawn Hochman \\ Department of Physiology, Emory University, Atlanta, Georgia 30322
}

\begin{abstract}
Spiking activity in motor axons represents the final central coding for muscle contraction. Recurrent collaterals in spinal cord from these same axons are known to offer a negative feedback control of motor output via a class of interposed inhibitory interneurons. Here we demonstrate that, during noradrenergic drive, a previously unknown recurrent excitatory pathway is unmasked and expressed. These excitatory projections are shown to have broad bilateral actions within and between hindlimb spinal segments and can alter ongoing pattern-generating motor behaviors. Thus, motor output strength is controlled via central positive and negative feedback loops, undoubtedly to provide a greater flexibility and dynamic range of control. That this novel function is regulated by a descending neuromodulatory transmitter indicates a conditional recruitment during certain behavioral states as part of the central noradrenergic arousal apparatus.
\end{abstract}

Key words: recurrent; excitation; motoneuron; collateral; glutamatergic; cholinergic

\section{Introduction}

Recurrent connectivity is a fundamental organizational principle found throughout the CNS, including neocortex, hippocampus, and basal ganglia (Schwartzkroin et al., 1990; Smith and Bolam, 1990; Martin, 2002), and can include both negative and positive feedback loops (Shu et al., 2003; Austin and Buckmaster, 2004). The ubiquity of recurrent connections emphasizes their importance in information processing throughout the nervous system. The spinal cord contains a well studied negative feedback loop regulating the firing of $\alpha$-motoneurons, the principal output neurons of the CNS encoding muscle contraction. Motoneuron axon collaterals within the spinal cord form excitatory connections onto inhibitory interneurons, named Renshaw cells, which in turn feedback onto and inhibit motoneurons (Renshaw, 1941; Eccles et al., 1954). In addition, there is also the potential for motor axon collaterals to recurrently excite motoneurons via motoneuron-to-motoneuron synapses, which have been identified anatomically in the adult cat hindlimb (Cullheim et al., 1977), as well as with electrophysiology in the newborn mouse (Nishimaru et al., 2005). Previously, there has not been evidence for recurrent excitation through interneuronal pathways.

The existence of recurrent excitatory feedback onto motoneurons would add an additional degree of control over motoneuron excitability. However, in developmentally mature mammals, the existence of recurrent excitatory actions remain both elusive and controversial, despite recent physiological evidence of monosynaptic motoneuron-to-motoneuron connections demonstrated in

Received 0ct. 28, 2005; revised April 18, 2006; accepted April 19, 2006.

This work was supported by National Institutes of Health Grant NS040893. We thank Mike Sawchuk and Maggie Hatcher for technical assistance. We also thank Pete Wenner for discussions while the work was in progress.

Correspondence should be addressed to Shawn Hochman, Department of Physiology, 615 Michael Street, Emory University, Atlanta, GA 30322. E-mail: shochma@emory.edu.

DOI:10.1523/JNEUROSCI.4623-05.2006

Copyright $\odot 2006$ Society for Neuroscience $\quad$ 0270-6474/06/255920-09\$15.00/0 the newborn mouse (Nishimaru et al., 2005). Even here, the functional significance and regulation of this monosynaptic pathway has yet to be explored. The ability of any recurrent excitatory connections to have significant effects during the recruitment of functionally relevant populations of motoneurons, such as those recruited during the monosynaptic "stretch" reflex, has not yet been demonstrated.

Here, we reveal increases in motoneuron excitability mediated by chemical synaptic transmission after stimulation of motor axon collaterals in a mammalian preparation with hyperpolarizing inhibitory transmission. Evidence is presented that the recurrent excitatory feedback is both glutamatergic and cholinergic, can be independent of activity in inhibitory neurons, and diverges to modify motor output over several spinal cord segments. Moreover, we demonstrate that motoneurons, previously thought to only make connections to inhibitory interneurons centrally, can access excitatory interneuronal pathways to influence the behavior of networks of interneurons that contribute to motor coordination. Importantly, we demonstrate that, during reflex-evoked recruitment of motoneuron populations, recurrent excitation is normally undetectable but becomes unmasked by noradrenaline (NA). This state-dependent unmasking of recurrent excitation may explain why identification of this pathway has remained recalcitrant.

The association between recurrent excitation and activation of noradrenergic neural circuits provides a framework from which to consider the possible broad behavioral significance of this pathway. The major adrenergic inputs to the spinal cord are activated during the "fight-or-flight" response (Aston-Jones et al., 1999), and, given the involvement of noradrenergic circuitry in arousal (Berridge and Waterhouse, 2003), one hypothesis is that these emergent pathways are expressed under urgent conditions, giving mammalian motoneurons a much broader control 

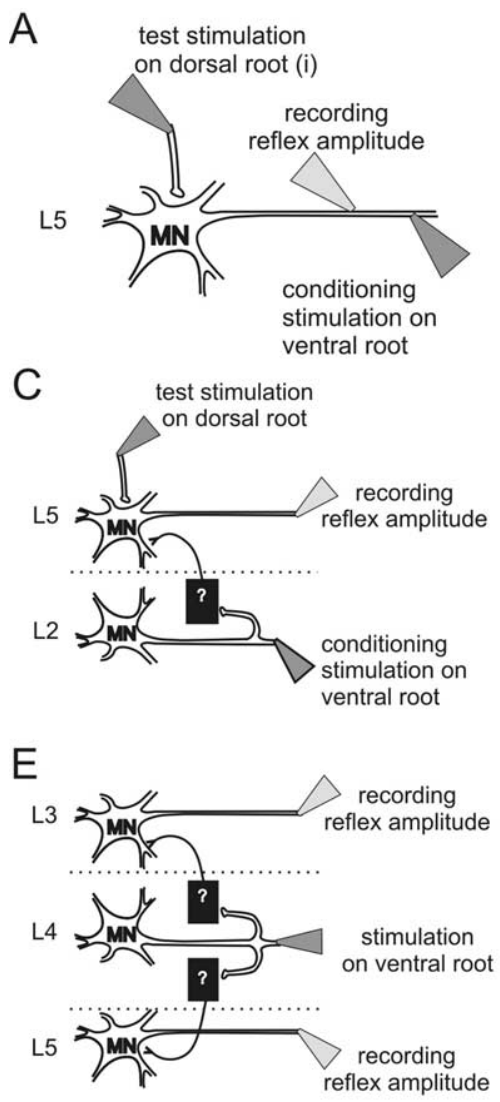

B

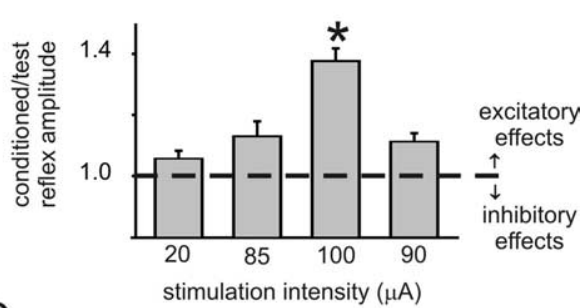

D

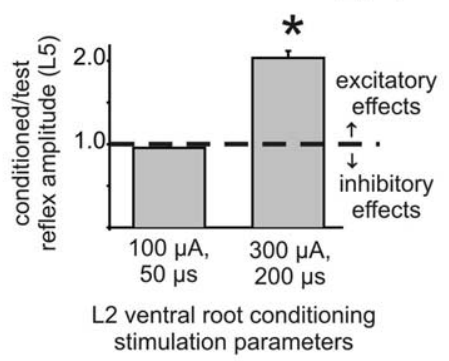

F

L4 VR stimulation

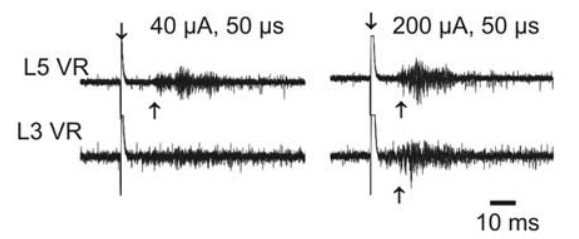

CSF (ACSF) containing the following (in $\mathrm{mm}$ ): $125 \mathrm{NaCl}, 2.5 \mathrm{KCl}, 1.25 \mathrm{NaH}_{2} \mathrm{PO}_{4}, 2 \mathrm{CaCl}_{2}, 1$ $\mathrm{MgCl}_{2}, 26 \mathrm{NaHCO}_{3}$, and 25 glucose, $\mathrm{pH} 7.4$ (equilibrated with $95 \% \mathrm{O}_{2} / 5 \% \mathrm{CO}_{2}$ ). The spinal cord was isolated and hemisected down the midline with an insect pin. The ACSF was gradually warmed to room temperature. The cord was stabilized with insect pins to the Sylgard bottom of the recording chamber. In some experiments, ACSF was perfused into the bath at a rate of $6 \mathrm{ml} / \mathrm{min}$; in others, a static bath was used.

Glass-suction electrodes were applied to dorsal and ventral lumbar roots. Constant-current stimuli were applied to roots while motor activity was recorded with a custom-built differential amplifier. Neurograms were amplified $(5000 \times)$ and low-pass filtered at $3 \mathrm{kHz}$. Highpass filtering ranged between 30 and $0.1 \mathrm{~Hz}$. Reflex amplitude was quantified by rectifying and integrating the ventral root recording (Machacek et al., 2001).

Experimental paradigm. Inhibitory transmitters are developmentally depolarizing but become hyperpolarizing onto motoneurons after P7 (Singer et al., 1998). To ensure that increases in excitability were not attributable to the release of depolarizing inhibitory transmitters, only animals older than $\mathrm{P} 7$ were used in this set of experiments.

In condition/test experiments, only the amplitude of first $3 \mathrm{~ms}$ of the monosynaptic reflex (defined as having a latency between 6 and 8 $\mathrm{ms}$ ) was examined because this is a known monosynaptic connection and any contaminating effects of conditioning on polysynaptic pathways could be negated. Test stimulation was delivered to the dorsal root [test reflex (TR)] of the same segment from which ventral root recording was made. Test stimulation intensity was adjusted such that it was approximately two times the threshold for a monosynaptic response with a test stimulus duration of $500 \mu$ s. Thirty seconds later, dorsal root stimulation was repeated, this time preceded $20 \mathrm{~ms}$ by ventral root stimulation, with intensities ranging from 20 to $500 \mu \mathrm{A}$ and duration ranging from 50 to $500 \mu \mathrm{s}$ [conditioned reflex (CR)]. Stimuli of $300 \mu \mathrm{A}$ and $100 \mu$ s or greater produced maximal ventral root volleys. Stimulating the ventral root antidromically activates motoneuron axons, thereby causing neurotransmitter release from their axon collaterals. Conditioning was either done within a lumbar segment (such that recording occurred in the same ventral root that was conditioned) (Fig. $1 A)$ or between different lumbar segments root (Fig. 1C).

The CR amplitude was normalized to the TR amplitude (the CR amplitude divided by the TR

of central circuits than previously imagined during certain behavioral states.

\section{Materials and Methods}

Preparation. Sprague Dawley rats postnatal day (P0) to P16 were anesthetized with urethane ( $2 \mathrm{mg} / \mathrm{kg}$, i.p.), decapitated, and eviscerated. For condition/test experiments described below, only rats P7 or older were used. The spinal column, with hindlimbs attached, was then placed in a bath dissecting chamber containing cooled $\left(4^{\circ} \mathrm{C}\right)$ oxygenated artificial amplitude). This was repeated for 25 reflex pairs, and the normalized values were averaged. If there was an inhibitory effect of conditioning, the normalized value of the $\mathrm{CR}$ would be less than 1 . Conversely, the value of the normalized CR would be greater than 1 for an excitatory effect with the conditioned reflex larger than the test reflex (Fig. $1 B$ ). Conditioning stimulation alone was tested and resulted in no motor output unless otherwise mentioned.

For studies of locomotor-like activity (LLA), P0-P3 animals were used with suction electrodes on the L2 and L5 ventral roots. Activity in these 
A

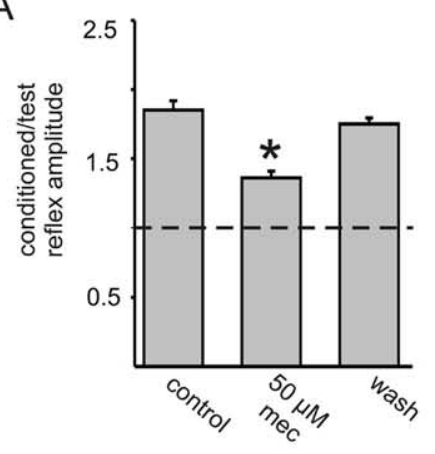

B

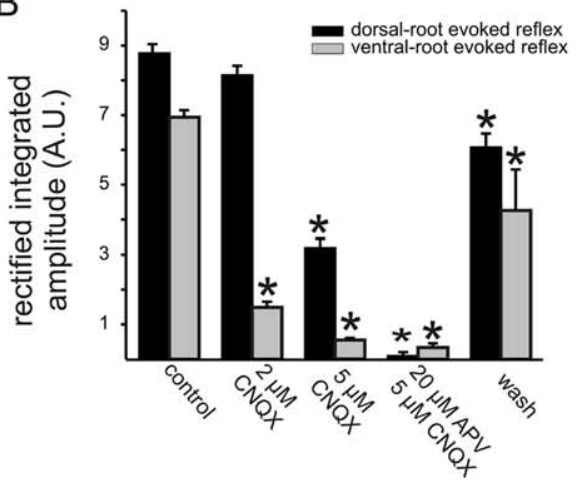

C

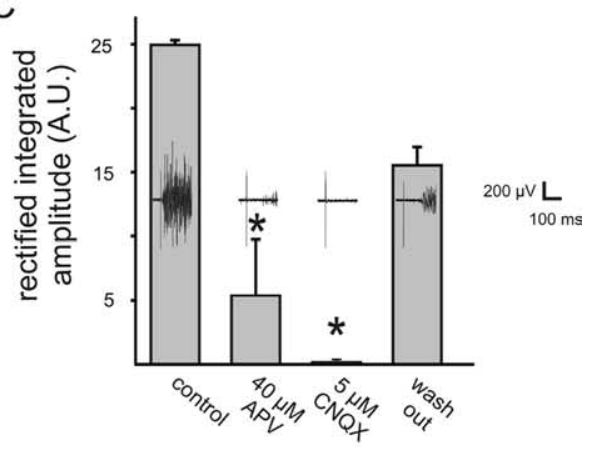

Figure 2. Excitatory effects from ventral root conditioning are sensitive to cholinergic and glutamatergic receptor antagonists. $A$, Addition of mecamylamine (mec) significantly decreased the recurrent excitatory effects of $L 5$ ventral root conditioning on an $L 5$ reflex $\left({ }^{*} p<0.05 ; n=25\right)$. $\boldsymbol{B}$, Essential role of AMPA/kainate receptors in the expression of VRERs. Drugs and applied concentrations are shown on the abscissa. Note that CNQX had a greater effect on the ventral-root-evoked but not the dorsal-root-evoked reflex. Peak amplitudes were measured from the first 100 ms after stimulus artifact in arbitrary units (A.U.) ( $\left.{ }^{*} p<0.05 ; n \geq 4\right)$. C, NMDA receptors contribute to the VRER. Similar results obtained in $\boldsymbol{B}$ except that the order of application of non-NMDA and NMDA receptor antagonists is reversed. Sample raw traces are shown within each treatment group. Ventral root reflexes were evoked by stimulation of $L 4$ at $150 \mu A, 50 \mu \mathrm{s}$, with an interval of $30 \mathrm{~s}$ between episodes shown for a given drug application. Note that, whereas high doses of the NMDA receptor antagonist APV did not completely block the ventral-root-evoked reflex, CNQX completely and reversibly blocked the evoked response $\left({ }^{*} p<0.05 ; n \geq 3\right.$ ). This experiment was conducted in the presence of bicuculline $(5 \mu \mathrm{M})$ to facilitate expression of ventral-root-evoked reflexes (see Fig. $3 A)$. Average background noise was subtracted from each of the signals in $\boldsymbol{B}$ and $\boldsymbol{C}$.

roots reflects motor activity in flexors and extensors, respectively (Kiehn and Kjaerulff, 1996).

Pharmacology. The following compounds were used: 5-HT (10-50 $\mu \mathrm{M})$, bicuculline methiodide $(5-20 \mu \mathrm{M})$, strychnine $(0.5-10 \mu \mathrm{M})$, mecamylamine $(50 \mu \mathrm{M})$, dihydro- $\beta$-erythroidine $(20 \mu \mathrm{M})$, d-tubocurarine $(10-80 \mu \mathrm{M})$, NA (10-50 $\mu \mathrm{M})$, kynurenic acid (1000 $\mu \mathrm{M}), 2$-amino-5-phosphonovaleric acid (APV) (20 $\mu \mathrm{M})$, and 6-cyano-7-nitroquinoxaline-2,3-dione (CNQX) $(10 \mu \mathrm{M})$. All drugs were obtained from Sigma-Aldrich (St. Louis, MO) and were applied in dissolved ACSF.

Whole-cell patch recording. Whole-cell "blind" patch-clamp recordings were made from hemisected spinal cords as described previously (Hochman and Schmidt, 1998) to record evoked postsynaptic potentials after stimulation of attached roots. Patch electrodes contained $140 \mathrm{~mm}$ K-gluconate, $11 \mathrm{~mm}$ of EGTA, $10 \mathrm{~mm}$ HEPES, $1 \mathrm{~mm}$ of $\mathrm{CaCl}_{2}$, and $35 \mathrm{~mm}$ $\mathrm{KOH}, \mathrm{pH} 7.3$, and resistances measured in ACSF ranged from 4 to 12 $\mathrm{M} \Omega$. For voltage-clamp recordings, K-gluconate was occasionally replaced with CsF. Neurobiotin (0.02\%; Vector Laboratories, Burlingame, CA) was also occasionally included in the patch electrodes. Recordings were obtained in conjunction with an Axopatch 1D amplifier (Molecular Devices, Union City, CA). Data were acquired and analyzed on a personal computer using pClamp software (version 9.0; Molecular Devices). Resting membrane potential, leak conductance, and compensated series resistance (bridge balance) were monitored throughout to ensure recording stability. Synaptic potentials were recorded at the same membrane potential before, during, and after drug application to ensure reliable comparison of changes in membrane potential.

Analysis. A paired $t$ test on the normalized value of the amplitude of the CR versus the amplitude of the TR was conducted to determine the significance of recurrent effects in control conditions. The effects of drugs were examined by comparing the normalized CR amplitudes using a repeated-measures ANOVA with a level of significance at $p=0.05$. Post hoc Dunnett's tests were performed to compare drug treatments with control. Normality was tested at a level of $p \leq 0.01$, and, if normality failed, an ANOVA was performed on ranks. All statistical computations were made using SigmaStat software, and data were plotted with SigmaPlot (both by SPSS, Chicago, IL). Graphical values are presented as mean \pm SEM.

Immunoprocessing. Spinal cords were removed and immersion fixed with $4 \%$ paraformaldehyde for $1 \mathrm{~h}$. The cords were subsequently cut into $10-\mu \mathrm{m}$-thick sections on a cryostat and processed for immunohistochemistry. Anti-calbindin D-28k (rabbit, 1:1000; Chemicon, Temecula, CA) was used. Immunoreactive sites were revealed with donkey antirabbit secondary antibody (Jackson ImmunoResearch, West Grove, PA) conjugated to a cyanine 3 fluorochrome. Neurobiotin-filled cells were revealed using streptavidin conjugated to an Alexa 350 fluorochrome (Invitrogen, Carlsbad, CA). Images were photographed with a Nikon (Tokyo, Japan) digital camera through a Nikon E800 microscope. Images were processed (contrast-enhanced and pseudocolored) to maximize quality and background staining of blood vessels subtracted using Corel Draw (Corel, Ottawa, Ontario, Canada).

\section{Results}

\section{Effects of ventral root stimulation}

We used the immature rat spinal cord maintained in vitro to characterize motor activity in a precisely controlled pharmacologic and ionic environment. To test the effects of ventral root stimulation (recurrent motor activity) on motoneuron excitability, we used the well established condition/test paradigm (Renshaw, 1941; Baldissera et al., 1981) in animals P7 and older (see Materials and Methods) (Fig. 1A). Briefly, changes in the amplitude of the dorsal root-evoked monosynaptic reflex (the test stimulus) were used to detect subthreshold actions in motoneurons from stimulation of motor axons in ventral roots (the conditioning stimulus). Using this approach, we observed that ventral root stimulation reduced motoneuron excitability 31 of 110 times, consistent with recruitment of the well known pathway of recurrent inhibition. Unexpectedly, excitatory effects were found in 20 of 110 experiments. Excitatory effects were observed both within (Fig. $1 B$ ) and between (Fig. $1 D$ ) lumbar segments, were dependent on stimulation intensity (Fig. $1 B, D$ ), and were usually subthreshold ( 12 of 20). However, in eight cases, no conditioning was required because ventral root stimulation alone recruited motoneuron firing, seen as a ventral-root-evoked reflex (VRER) (Fig. 1F). VRERs were mediated by $\mathrm{Ca}^{2+}$-dependent chemical synaptic transmission because changing the bath solution to a nominally $\mathrm{Ca}^{2+}$-free saline reversibly blocked evoked activity $(n=3)$ (Fig. 1G). To further support chemical synaptic transmission, in two of three cases, the nonspecific gap junction blocker carbenoxolone $(100 \mu \mathrm{M})$ did not block excitatory effects of conditioning. This demonstrates that gap-junctional coupling is not required for recurrent excitatory effects in these cases (data not illustrated).

VRERs elicited from stimulation of the same lumbar segment as recorded had, on average, a latency $5 \mathrm{~ms}$ longer than a monosynaptic reflex $(12 \pm 1.1 \mathrm{~ms}$ compared with $7.2 \pm 0.3 \mathrm{~ms}$, respec- 
A

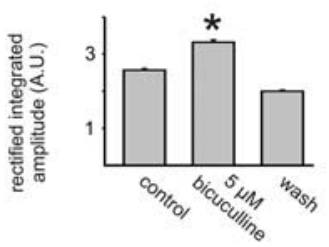

C L5 VR stimulation

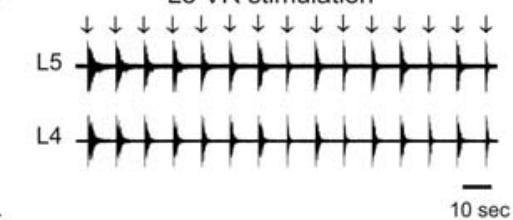

$\mathrm{E}$

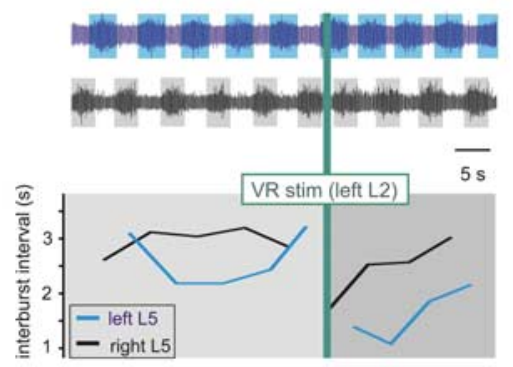

B

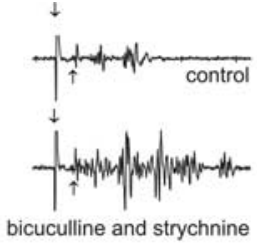

D

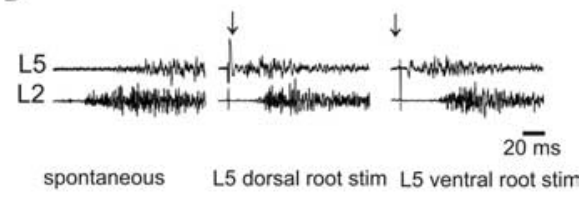

$\mathrm{F}$

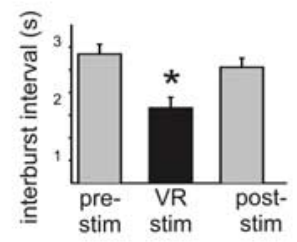

G

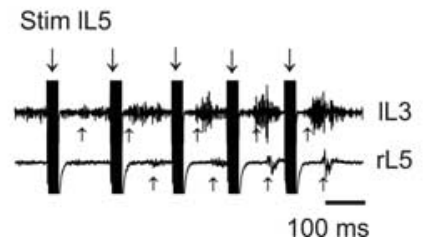

Figure 3. Effects of blocking inhibitory transmission on recurrent excitation. $A$, VRER amplitude in arbitrary units (A.U.) was reversibly increased after application of $5 \mu \mathrm{m}$ bicuculline $\left.{ }^{*} p<0.05 ; n=25\right)$. $\boldsymbol{B}$, Blockade of inhibitory transmission with bicuculline $(20 \mu \mathrm{M})$ and strychnine $(10 \mu \mathrm{M})$ increased ventral-root-stimulation-evoked reflex amplitude and duration. Stimulus intensity was $150 \mu \mathrm{A}, 50 \mu \mathrm{s}$. C, Recurrent excitatory pathways can entrain convulsant-evoked motor activity. In the presence of bicuculline $(10 \mu \mathrm{M})$ and strychnine $(0.5 \mu \mathrm{M})$, spontaneous synchronous bursting is seen in both $L 4$ and $L 5$ ventral roots (VR) (not illustrated). Similar bursts could be reliably initiated and entrained by $L 5$ ventral root stimulation (stimulus intensity of $300 \mu A$, $200 \mu S)$. D, Recurrent excitatory pathways can alter initiation site of motor bursts. Spontaneous bursting appeared first on the L2 ventral root and then in the $L 5$ ventral root $\sim 15$ ms later (left panel). However, stimulation of $L 5$ dorsal or ventral root could entrain the bursting to initiate on the $L 5$ segment $\sim 20$ ms before the onset of $L 2$ bursting activity (middle and right panels). $\boldsymbol{E}, \boldsymbol{F}$, Recurrent excitatory pathways increase locomotor frequency. The effects of ventral root stimulation on 5-HT and NMDA-induced locomotor-like activity $(\boldsymbol{E})$ in the presence of NA $(100 \mu \mathrm{M})$. Raw (top) and corresponding interburst interval (bottom) before, during, and after a single train of stimulation to the left $L 2$ ventral root $(20$ stimuli at $50 \mathrm{~Hz}) . F$, In a separate experiment, the mean interburst interval for 10 bursts of locomotion are plotted. Ventral root stimulation was applied in trains (10 stimuli at $333 \mathrm{~Hz}$ ) to the L2 ventral root in the presence of NA $(100 \mu \mathrm{M})$ and mecamylamine $(50 \mu \mathrm{m})$ immediately at the termination of each extension phase burst of locomotor-like activity. The decrease in intraburst interval demonstrates an increased frequency ${ }^{*} p<0.05 ; n=$ 10). $G$, In this animal, just after locomotor activity stopped, the same ventral root stimulus (trains of 10 stimuli at $333 \mathrm{~Hz}$ ) was able to recruit motor activity bilaterally, indicating an interaction with commissural interneurons. IL5, IL3, and rL5 denote left L5, left $\mathrm{L} 3$, and right $\mathrm{L} 5$ ventral roots.

tively; $p<0.05 ; n=9)$, with more variation in latency. Because synaptic delay at room temperature is $\sim 2.5-3.0 \mathrm{~ms}$ (Jonas et al., 1998), the average latency of the recurrent reflex is consistent with a disynaptic connection. However, the minimum latency seen was $7.1 \mathrm{~ms}$, which could be explained by the occasional additional recruitment of a monosynaptic, motoneuron-tomotoneuron input (Cullheim et al., 1977; Nishimaru et al., 2005).

\section{Pharmacology of recurrent excitatory circuitry}

Because motoneurons are cholinergic, effects mediated via recurrent collaterals would be expected to demonstrate sensitivity to cholinergic receptor antagonists. The cholinergic receptor antagonists mecamylamine $(n=4)$ (Fig. $2 A)$ and dihydro- $\beta$ erythroidine (data not shown; $n=2$ ) reversibly decreased the excitatory effects of conditioning but never completely blocked them. In comparison, VRERs could be blocked by ionotropic glutamate receptor antagonists. CNQX $(2-5 \mu \mathrm{M})$, an AMPA/kai- nate receptor antagonist, completely blocked the VRER three of five times (Fig. $2 B)$. The NMDA receptor antagonist APV $(20-50 \mu \mathrm{M})$ decreased reflex amplitude four of five times but never completely blocked VRERs, even at high doses (Fig. $2 C)$. The nonspecific ionotropic glutamatergic receptor antagonist kynurenic acid (1 mM) also blocked the VRER two of two times (data not shown). VRERs resistant to $\mathrm{CNQX}$ were subsequently blocked with cholinergic antagonists $(n=2$ of 2$)$. Consistent with recent observations (Mentis et al., 2005; Nishimaru et al., 2005), we conclude that the recurrent excitatory pathway is partially mediated by both cholinergic and AMPA/kainate/NMDA receptor-dependent glutamatergic transmission.

\section{Effects of ventral root simulation in the} absence of inhibitory transmission In three of five experiments without previous evidence of recurrent excitation, partial block of $\mathrm{GABA}_{\mathrm{A}}$ receptors with bicuculline $(5 \mu \mathrm{M})$ brought out excitatory effects. The inability to observe recurrent excitation in the other two experiments suggest that additional mechanisms also control or limit access to this pathway. Moreover, in three of four cases with evoked VRERs, bicuculline $(5-10 \mu \mathrm{M})$ caused both an increase in reflex amplitude and duration without changing onset latency (Fig. 3A). These observations further demonstrate that GABAergic activity limits recurrent excitatory actions. Because glycinergic inhibition also acts on spinal motor circuits, we examined recurrent actions after block of both $\mathrm{GABA}_{\mathrm{A}}$ and glycine receptors with a combination of bicuculline $(10-20 \mu \mathrm{M})$ and strychnine $(0.5-10 \mu \mathrm{M})$, respectively. Once again, recurrent excitatory actions were facilitated $(n=3)$ (Fig. 3B). These results also demonstrate that actions from inhibitory circuits are not required for the expression of recurrent excitatory actions.

As demonstrated previously, blockade of GABAergic and glycinergic inhibitory transmission results in the emergence of spontaneous synchronous bursting in motor pools from all recorded ventral roots (Bracci et al., 1996). We examined whether activating recurrent collaterals could affect this bursting but were unable to detect any changes in animals younger than P9 $(n=$ 10). However, in two of nine experiments on animals aged P11P14, ventral root stimulation entrained the bursting episode (Fig. $3 C)$. After addition of 50-100 $\mu \mathrm{M} \mathrm{NA}$, ventral root stimulation entrained bursting in an additional animal for a total of three of nine experiments. In an additional case, spontaneous bursting was obtained by addition of bicuculline $(20 \mu \mathrm{M})$ alone, and this bursting was entrained by ventral root stimuli, resulting in a total of 4 of 10 P11-P14 animals having entrainable bursting via recurrent excitatory pathways. Interestingly, both sensory input (dor- 
A

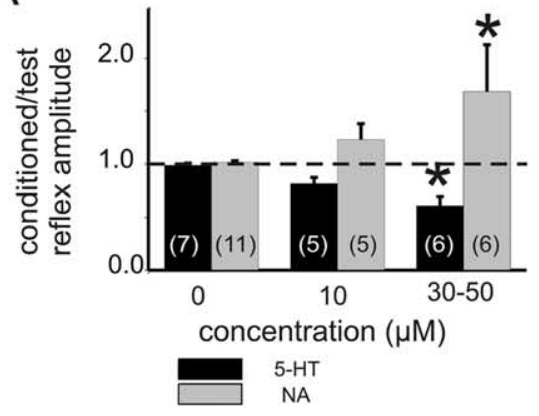

B

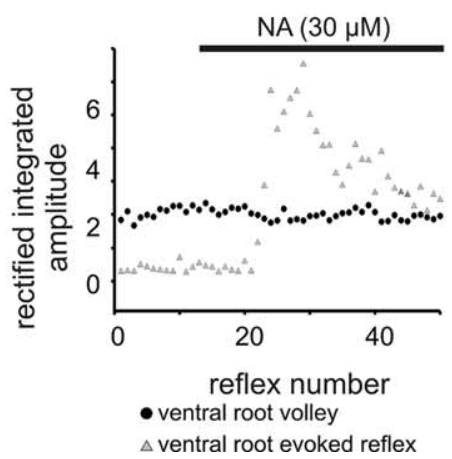

C

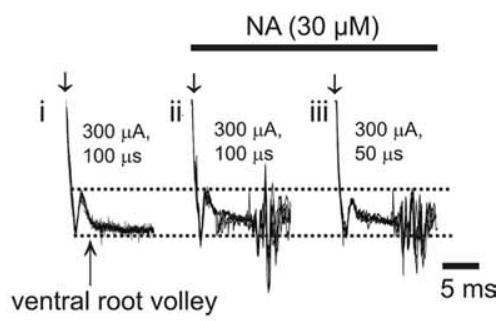

Figure 4. NA unmasks recurrent excitatory actions. A, In experiments without detectable recurrent excitation, either 5-HT or NA was then applied at 10 or $30-50 \mu$ m. Bars represent the mean response between animals (sample size shown bracketed in histogram), and results obtained for each animal were the average of 25 reflex pairs per condition. Note that NA unmasked recurrent excitatory and 5-HT unmasked recurrent inhibitory actions in a concentration-dependent manner $\left({ }^{*} p<0.05\right)$. $\boldsymbol{B}$, Comparison of amplitude of rectified/integrated ventral root volley (a measure of direct motor axon recruitment) to that of an evoked reflex. Note that the NA-evoked unmasking of the VRER occurs in the absence of changes motor axon recruitment, demonstrating that the induced recurrent excitation occurs by modulatory actions in spinal cord. C, Raw superimposed traces of evoked events at $L 5$ after stimulation of the same root at intensities shown. $i$, Under control conditions, no reflex is evoked but the compound action potential representing direct motor axon recruitment is identified as an incoming ventral root volley (arrow). Note that, compared with control conditions (i), NA allows expression of an evoked reflex (ii) that persists even when the stimulation duration has been lowered (iii). Horizontal dashed lines demarcate the amplitude of the ventral root volley in control conditions to facilitate comparison between conditions.

sal root) and motor axon collaterals (ventral root) could initiate local onset epileptiform bursting episodes that would have otherwise begun at more distant foci (Fig. 3D). This suggests that afferents and motor axon collaterals converge onto common excitatory pathways. Thus, in the absence of spinal inhibition, recurrent motor axon collaterals can feedback onto excitatory pathways across multiple spinal segments, but this pathway appears to be commonly inaccessible in our preparation. Indeed, in two of three experiments in which bursting was entrained by ventral root stimulation, the ability to entrain activity was lost over time, suggesting a transient access or fatigable circuitry. Because spontaneous bursting could be entrained by dorsal root stimuli in 6 of 10 experiments in which ventral root stimuli could not entrain bursts, the ventral root excitatory pathway is clearly not necessary for spontaneous bursting to occur (cf. Bracci et al., 1997). Importantly, in some cases, ventral root stimulation could not entrain spontaneous bursting yet evoked ventral root reflexes $(n=3)$, demonstrating that access to at least some of the interneuronal circuitries involved in spontaneous bursting can be independent of those producing recurrent excitation.

\section{Recurrent excitatory pathways reinforce an ongoing motor behavior}

The spinal cord contains all of the neuronal circuitry for producing fictive locomotion (Brown, 1911; Grillner, 1981; Kiehn and Kjaerulff, 1998). In the neonatal rat, addition of 5-HT and NMDA can produce a rhythmic ventral root activity consistent with locomotion (Cazalets et al., 1992; Kiehn and Kjaerulff, 1996). It was shown recently that in the mouse, ventral root stimulation could initiate locomotor-like activity (Mentis et al., 2005), but the ability of ventral root stimulation to modify ongoing pattern generation while it was active has not been investigated. To determine whether recurrent motor axon collaterals had an influence on the locomotor circuitry, we tested the effects of trains of ventral root stimulation during 5-HT/NMDA-evoked LLA at $\mathrm{P} 0-\mathrm{P} 3$. We found that ventral root stimulation was unable to modify LLA in all six animals tested. However, in the presence of NA $(50-100 \mu \mathrm{M})$, ventral root stimuli increased locomotor frequency in two of three cases (Fig. $3 E, F$ ), including once in the presence of $50 \mu \mathrm{M}$ mecamylamine. These actions are consistent with the demonstrated importance of NA in reinforcement and maintenance of locomotor activity (Kiehn et al., 1999) and suggest that an adrenergic unmasking of transmission through recurrent excitatory pathways can contribute an excitatory drive to the locomotor rhythm. Importantly, during this series of experiments, trains of stimuli to the ventral roots were also capable of producing bilateral VRERs (Fig. 3G). Because lumbar Renshaw cells and motoneurons do not project contralaterally, other interneurons must be involved in these responses.

\section{NA and 5-HT have opposing actions on recurrent circuitry}

As reported previously, recurrent excitation was observed in only $18 \%$ (20 of 110) of the experiments. In cases in which ventral root stimulation did not facilitate test reflex-evoked motor activity, moderate doses of NA $(10 \mu \mathrm{M})$ unmasked recurrent excitation in two of five cases, whereas higher doses $(30-50 \mu \mathrm{M})$ more consistently recruited recurrent excitation $(n=5$ of 6$)$ (Fig. $4 A)$. The unmasking effects of NA occurred without changing the amplitude of the ventral root spike volley, indicating a spinal site of modulation (Fig. $4 B$ ), not a change in the number of axons recruited. Unmasked recurrent excitation did not require maximal motor axon recruitment (Fig. $4 C$ ). In contrast to NA, application of 5-HT $(50 \mu \mathrm{M})$ never brought out recurrent excitatory actions and instead promoted recurrent inhibitory action in six of six of these animals (Fig. 4A). Thus, of the two predominant descending monoaminergic transmitters, NA, supported the emergence of recurrent excitation, whereas 5-HT favored recurrent inhibitory actions.

\section{Identification of neurons in recurrent pathways}

Although it is clear that motoneurons are first-order neurons in recurrent excitatory pathways, the observed widespread excitatory actions implicate the existence of additional interposed neurons, at least some of which are gated by NA. Currently, only Renshaw cells and motoneurons have been shown to receive recurrent input from motoneuron axon collaterals (Cullheim et al., 1977; Baldissera et al., 1981; Nishimaru et al., 2005).

The purpose of this study was to report the existence of previously unknown recurrent excitatory actions on motor output. Nonetheless, we also felt it important to strengthen these observations with the identification of some candidate interneurons. Fifty-four whole-cell recordings were performed examining the 
A

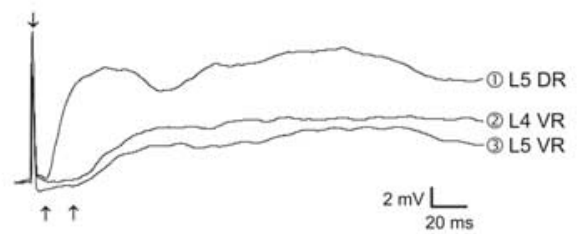

C

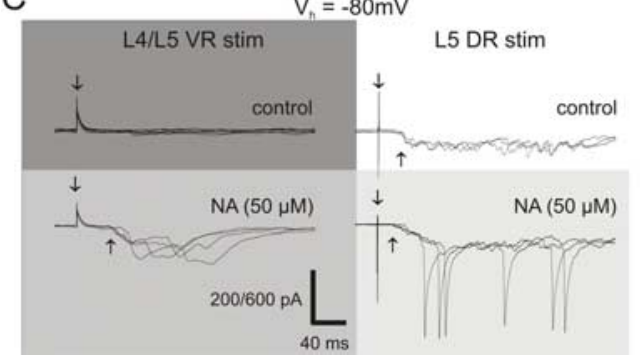

B

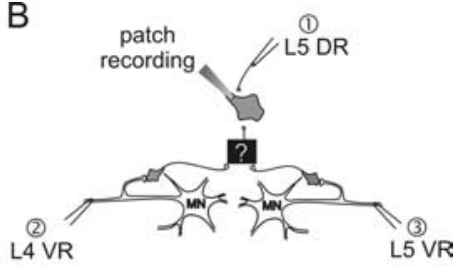

D

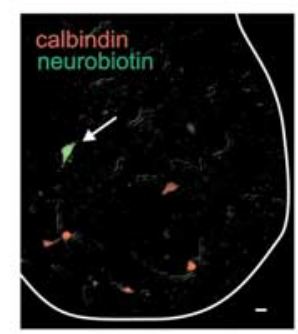

Figure 5. Evidence for the existence of interneurons having actions expected from the properties of the evoked reflexes. $\boldsymbol{A}$, Average of 10 whole-cell current-clamp recordings of a cell in the ventral horn near the motor pool that received short-latency excitation from dorsal root stimulation ( $500 \mu \mathrm{A}, 500 \mu \mathrm{s})$ and long-latency excitation from ventral root stimulation (400 $\mu \mathrm{A}, 200$ $\mu s)$. $B$, Schematic diagram of the experimental setup illustrating the respective stimulating sites for responses (1-3) and hypothetical circuitry consistent with the observed actions. C, NA-dependent ventral-root-evoked synaptic actions in a spinal neuron. Membrane voltage was clamped at a holding potential of $-80 \mathrm{mV}\left(V_{h}\right.$ of $\left.-80 \mathrm{mV}\right)$. Under control conditions, the neuron received long-latency sensory input ( $300 \mu \mathrm{A}, 200 \mu \mathrm{s})$ but no input from simultaneous L4/L5 ventral roots (top row; $300 \mu \mathrm{A}, 200 \mu \mathrm{s})$. After addition of NA (50 $\mu \mathrm{m})$, sensory input was facilitated and long-latency synaptic inputs after VR stimulation were unmasked (bottom row). The vertical scale bar represents $200 \mathrm{pA}$, except for the trial with dorsal root stimulation in the presence of NA in which it represents 600 pA. D. The neuron in C was Neurobiotin filled to identify its location (white arrow). Both the location and lack of calbindin immunostaining (a marker of Renshaw cells) demonstrate that the recorded neuron is not a Renshaw cell. Scale bar, $25 \mu \mathrm{m}$. DR, Dorsal root; VR, ventral root.

effects of ventral root stimulation in ventral horn neurons, and two neurons with previously unreported recurrent input properties were encountered. We identified a neuron near the L5 motor pool in a P7 animal having synaptic inputs consistent with a polysynaptic excitation from motoneurons (Fig. 5A). Its high membrane resistance [510 $\mathrm{M} \Omega$ (cf. Hochman and Schmidt, 1998)] and lack of antidromic response support an interneuronal identity. This cell received long-latency synaptic input ( $23 \mathrm{~ms}$ ) from both L4 and L5 ventral roots that was partly blocked by mecamylamine and short-latency input from the L5 dorsal root (7 ms compared with a $6.5 \mathrm{~ms}$ monosynaptic reflex latency). The lack of monosynaptic input from ventral roots precludes Renshaw cell identity and confirms the existence of interneurons that receive polysynaptic input from motoneuron axon collaterals. We also identified an interneuron that received sensory input but no response after ventral root stimuli. Interestingly, after application of NA, long-latency ventral root stimulation-evoked synaptic input was unmasked (Fig. 5C) and sensory input responses were facilitated. This interneuron was filled with Neurobiotin, and immunoprocessing demonstrated that it was calbindin negative and dorsomedial to motor pools (Fig. 5D), inconsistent with identification as a Renshaw cell (Carr et al., 1998). Thus, additional neurons, other than motoneurons or Renshaw cells, also receive input from motoneuron collaterals.

\section{Discussion}

We demonstrated that activity in motor axon collaterals can feedback onto spinal neural circuits and lead to increases in motoneuron excitability mediated by chemical synaptic transmission. Recurrent excitatory feedback is sensitive to cholinergic and glutamatergic receptor antagonists and can be recruited in the absence of inhibitory transmission. The excitatory pathways in-

volve interneurons because they diverge to modify output over several spinal cord segments bilaterally. Recurrent projections access interneurons that contribute to motor coordination because they can entrain epileptiform activity and facilitate ongoing locomotion. Importantly, recurrent excitation is normally undetectable but becomes unmasked by NA. Thus, positive feedback is a state-dependent behavioral feature of the motor system recruited during activation of descending adrenergic circuits.

Recurrent facilitation was first observed in cat (Renshaw, 1941) and has subsequently been attributed to a disinhibition by the action of Renshaw cells on other tonically active inhibitory interneurons (other Renshaw cells and Ia inhibitory interneurons) (Wilson and Burgess, 1962; Hultborn et al., 1971; McCrea et al., 1980; McCurdy and Hamm, 1994). This explanation is consistent with known circuitry, and, in some cases, recurrent facilitation is clearly the result of disinhibition. Our data necessitate novel circuitry because we observed recurrent excitation after blockade of inhibitory transmission. Furthermore, the unmasked long-latency responses diverge to several spinal segments bilaterally, which is inconsistent with the known projections of motoneuron recurrent collaterals or Renshaw cells (Baldissera et al., 1981).

A caveat of the current work is the report of many observations based on effects demonstrated from small numbers of experiments. In many cases, this is related to the characterization of pathways expressed in a small minority of experiments. Hence, additional work is required to provide a more detailed appraisal of this initial identification of novel recurrent excitatory pathways.

\section{Recurrent excitation and glutamatergic transmission}

Previous studies on the in vitro rodent spinal cord have identified recurrent excitatory actions in motoneurons partially mediated by glutamatergic transmission (Jiang et al., 1991; Schneider and Fyffe, 1992; Ichinose and Miyata, 1998; Nishimaru et al., 2005). Because motoneurons are traditionally considered cholinergic neurons, one study attributed glutamatergic actions to activation of sensory afferent axons located in ventral roots (Jiang et al., 1991). Although unmyelinated afferents can be found in ventral motor roots associated with autonomic spinal segments (Coggeshall, 1980), there is no anatomical evidence for spinal cord entry through spinal segments primarily involved in motor control, including the lumbar enlargement (for review, see Hildebrand et al., 1997). Conversely, there is now strong evidence that mammalian motoneurons release glutamate at synaptic terminals (Ichinose and Miyata, 1998; Malomouzh et al., 2003; Herzog et al., 2004; Kraus et al., 2004) with demonstrated glutamatergic actions on Renshaw cells and motoneurons (Mentis et al., 2005; Nishimaru et al., 2005).

A previous study reported ventral-root-evoked reflexes (Nishimaru et al., 2005) in animals in which inhibitory transmitters are still depolarizing and hence could be explained by the 
actions of Renshaw cells on motoneurons. Presently, in 7- to 16-d-old rats in which response to inhibitory transmitters should be hyperpolarizing (Singer et al., 1998), the mean latency of ventral-root-evoked reflexes was $\sim 5 \mathrm{~ms}$ longer, and more variable in onset, than the monosynaptic reflex evoked with dorsal root stimulation. Synaptic delay at room temperature is $\sim 2.5-3.0$ ms (Jonas et al., 1998), so the average latency of the recurrent reflex is consistent with a disynaptic pathway. Previous studies examining the excitatory response to ventral root stimulation with intracellular recordings of motoneurons have reported latencies the same or longer, with more variability in the latency than they report for the known disynaptic recurrent inhibitory responses, inconsistent with a monosynaptic connection (Jiang et al., 1991; Schneider and Fyffe, 1992; Ichinose and Miyata, 1998).

Minimally disynaptic excitatory connections require that motor axons synapse onto previously unidentified interneurons. Anatomic studies suggested that motor axons may synapse onto other neurons within lamina IX (Cullheim et al., 1977), the location of motoneurons and other interneurons (Alstermark and Kümmel, 1990). The present work identified other neurons with properties consistent with their involvement in longer-latency recurrent excitatory actions. First, we identified an interneuron receiving recurrent excitatory input only after application of NA. This supports an interneuronal mechanism involved in the NAinduced unmasking of recurrent excitation observed in five of six animals. Second, consistent with observations of longer-latency multisegmental recurrent excitatory actions on motor output, we also identified an interneuron that received excitatory polysynaptic connections from activation of multiple ventral roots and short-latency input from primary afferents. The convergence of afferent inputs on both cell types reported above suggests that interneurons not only relay feedback information from motoneurons but also respond to and integrate sensory information.

In addition to recurrent excitatory actions via interposed interneurons, monosynaptic excitation of motoneurons, as anatomically identified by Cullheim et al. (1977) and physiologically verified by Nishimaru et al. (2005), may also be recruited in some of our recurrent excitatory events. Moreover, because motoneurons also contain dendrodendritic synapses (Anderson et al., 1976), we cannot rule out the possibility that divergence of recurrent excitation via motoneuron connections is widespread.

\section{Synchronous bursting and locomotor network operation}

Blockade of synaptic inhibition can induce spontaneous epileptiform bursting in several CNS regions, including the cortex, hippocampus, and spinal cord (Hablitz, 1984; Hwa and Avoli, 1991; Bracci et al., 1996). Because cortex and hippocampus both have recurrent excitatory connections, we investigated whether recurrent excitatory pathways are also involved in spontaneous bursting activity in this preparation. Although stimulation of ventral roots did not entrain spinal epileptiform bursting activity in a previous study (Bracci et al., 1997), various conditions, including ventral root stimulus strength, may have differed from those reported here. Presently, bursting could be entrained by ventral root stimuli in P11-P14 (4 of 10) but not in younger ( 0 of 10 ) animals. Once opened, we demonstrated that recurrent pathways can tie into, but are not necessary for, the premotoneuronal network that coordinates paroxysmal bursting. A recent study in the mouse demonstrated that ventral root stimulation could elicit locomotor-like activity (Mentis et al., 2005). Here we add to the evidence that motoneuron activity can alter pattern generation by showing evidence that the frequency but not the pattern of ongoing rhythm was altered by ventral root stimula-

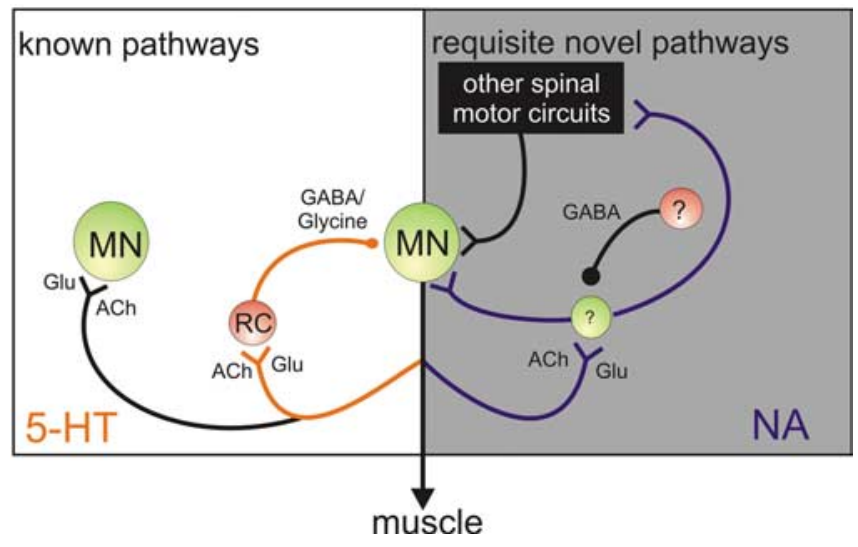

Figure 6. Simplified schematic diagram of known and putative recurrent spinal circuits. Left, Recurrent motor axon collaterals are known to project to motoneurons (MN) and Renshaw cells (RC). The present results demonstrate that recurrent inhibitory actions are facilitated by 5 -HT (orange). Right, Results from the present studies demonstrate that there must also be a group of excitatory interneurons responsible for minimally disynaptic recurrent excitatory connections onto motoneurons and actions on other interneurons that allow for a broader divergence of actions, including onto motor-pattern-generating networks. These excitatory pathways (blue) are inhibited by unidentified GABAergic interneurons and facilitated by noradrenaline (NA). Current evidence from several studies suggest that synaptic transmission from motor axon collaterals has cholinergic and glutamatergic receptor-dependent components, but whether this occurs through corelease at the same terminal or at separate synaptic terminals remains unclear. Glu, Glutamate; ACh, acetylcholine.

tion. Because we found significant effects only in the presence of NA, involvement of the recurrent excitatory pathway is implicated as opposed to a contribution from depolarizing inhibitory transmission.

\section{Monoaminergic control of recurrent feedback}

Factors determining whether recurrent excitation or inhibition is observed are uncertain but may relate to the animal's behavioral state. For example, recurrent inhibition is reduced during fictive locomotor activity in the cat (McCrea et al., 1980). Regarding behavioral state, the most important observation of the present study is that recurrent pathways are under monoaminergic control. The incidence of recurrent excitation increased from $18 \%$ (20 of 110) in the absence of NA to 83\% (5 of 6) in its presence. That recurrent excitation occurred in a minority of experiments in the absence of NA supports its elusive nature experimentally. The effects of NA included unmasking recurrent excitatory actions on spinal locomotor circuits. In this regard, it is of interest that NA has been reported to reinforce and maintain locomotor activity in this preparation (Kiehn et al., 1999), and NA release is thought to be important in locomotor initiation in the cat (Anden et al., 1966; Giroux et al., 2001). Interestingly, in contrast to NA, 5-HT promoted the expression of recurrent inhibition in all animals tested. That 5-HT and NA can have opposite actions on spinal circuits is consistent with the studies on identified interneurons by Jankowska et al. (2000).

\section{NA and the role of the locus ceruleus on motor behavior}

We identified a novel excitatory recurrent pathway regulated by NA that can significantly affect interneuronal spinal networks as well as regulate the excitability of the final common pathway of all CNS motor commands. Presumably, these modulatory actions would normally be recruited by cerulospinal activity because the principal sources of noradrenergic input to the spinal cord ventral horn arise from cerulospinal projections (Moore and Card, 
1984). Locus ceruleus circuitry is intimately associated with autonomic circuitry and is viewed as a "global rapid response system" that works in concert with sympathetic systems to produce "adaptive responses to urgent stimuli" (Aston-Jones et al., 1999). Thus, the facilitated motor activity reported here may be an integral part of a coordinated autonomic response, which could be particularly relevant during fight-or-flight behaviors.

\section{Conclusion}

Recurrent inhibitory actions on motoneurons can be altered by supraspinal systems (Baldissera et al., 1981), and it seems likely that similar control is available to recurrent excitatory pathways. Hultborn et al. (1979) put forward the "variable gain regulator" hypothesis, suggesting that facilitation of Renshaw cell inhibitory output would lower the input/output gain of the motoneuron pool to allow more finely tuned control by supraspinal centers (Hultborn et al., 1979). The recurrent excitation reported here would provide additional control for supraspinal centers to alter motor gain. A schematic of the proposed circuitry is illustrated in Figure 6, with the proposed novel NA responsive circuits presented on the right. Future studies are required to further characterize control of these circuitries, including whether recurrent inhibitory and excitatory inputs can be coactivated. The gain of cortical networks can be modulated by simultaneous increases in both excitatory and inhibitory "background" synaptic input mediated by recurrent excitatory and inhibitory circuits (Chance et al., 2002; Shu et al., 2003), and this mechanism may be recapitulated in spinal circuitry.

\section{References}

Alstermark B, Kümmel H (1990) Transneuronal transport of wheat germ agglutinin conjugated horseradish peroxidase into last order spinal interneurones projecting to acromio- and spinodeltoideus motoneurones in the cat. 1. Location of labelled interneurones and influence of synaptic activity on the transneuronal transport. Exp Brain Res 80:83-95.

Anden NE, Jukes MG, Lundberg A (1966) The effect of DOPA on the spinal cord. 2. A pharmacological analysis. Acta Physiol Scand 67:387-397.

Anderson WJ, Stromberg MW, Hinsman EJ (1976) Morphological characteristics of dendrite bundles in the lumbar spinal cord of the rat. Brain Res 110:215-227.

Aston-Jones G, Shipley MT, Grzanna R (1999) The locus coeruleus, A5 and A7 noradrenergic cell groups. In: The rat nervous system (Paxinos G, ed), pp 183-213. San Diego: Academic.

Austin JE, Buckmaster PS (2004) Recurrent excitation of granule cells with basal dendrites and low interneuron density and inhibitory postsynaptic current frequency in the dentate gyrus of macaque monkeys. J Comp Neurol 476:205-218.

Baldissera F, Hultborn H, Illert M (1981) Integration in spinal neuronal systems. In: Handbook of physiology, Sec I, The nervous system, pp 509595. Baltimore: Williams and Wilkins.

Berridge CW, Waterhouse BD (2003) The locus coeruleus-noradrenergic system: modulation of behavioral state and state-dependent cognitive processes. Brain Res Brain Res Rev 42:33-84.

Bracci E, Ballerini L, Nistri A (1996) Spontaneous rhythmic bursts induced by pharmacological block of inhibition in lumbar motoneurons of the neonatal rat spinal cord. J Neurophysiol 75:640-647.

Bracci E, Beato M, Nistri A (1997) Afferent inputs modulate the activity of a rhythmic burst generator in the rat disinhibited spinal cord in vitro. J Neurophysiol 77:3157-3167.

Brown GT (1911) The intrinsic factors in the act of progression in the mammal. Proc R Soc Lond B Biol Sci 84:308-319.

Carr PA, Alvarez FJ, Leman EA, Fyffe REW (1998) Calbindin D28k expression in immunohistochemically identified Renshaw cells. NeuroReport 9:2657-2661.

Cazalets JR, Sqalli-Houssaini Y, Clarac F (1992) Activation of the central pattern generators for locomotion by serotonin and excitatory amino acids in neonatal rat. J Physiol (Lond) 455:187-204.
Chance FS, Abbott LF, Reyes AD (2002) Gain modulation from background synaptic input. Neuron 35:773-782.

Coggeshall RE (1980) Law of separation of function of the spinal roots. Physiol Rev 60:716-755.

Cullheim S, Kellerth JO, Conradi S (1977) Evidence for direct synaptic interconnections between cat spinal alpha-motoneurons via the recurrent axon collaterals: a morphological study using intracellular injection of horseradish peroxidase. Brain Res 132:1-10.

Eccles JC, Fatt P, Koketsu K (1954) Cholinergic and inhibitory synapses in a pathway from motor-axon collaterals to motoneurones. J Physiol (Lond) 126:524-562.

Giroux N, Reader TA, Rossignol S (2001) Comparison of the effect of intrathecal administration of clonidine and yohimbine on the locomotion of intact and spinal cats. J Neurophysiol 85:2516-2536.

Grillner S (1981) Control of locomotion in bipeds, tetrapods, and fish. In: Handbook of physiology, The nervous system II, pp 1179-1236. Baltimore: Williams and Wilkins.

Hablitz JJ (1984) Picrotoxin-induced epileptiform activity in hippocampus: role of endogenous versus synaptic factors. J Neurophysiol 51:1011-1027.

Herzog E, Landry M, Buhler E, Bouali-Benazzouz R, Legay C, Henderson CE, Nagy F, Dreyfus P, Giros B, el Mestikawy S (2004) Expression of vesicular glutamate transporters, VGLUT1 and VGLUT2, in cholinergic spinal motoneurons. Eur J Neurosci 20:1752-1760.

Hildebrand C, Karlsson M, Risling M (1997) Ganglionic axons in motor roots and pia mater. Prog Neurobiol 51:89-128.

Hochman S, Schmidt BJ (1998) Whole cell recordings of lumbar motoneurons during locomotor like activity in the in vitro neonatal rat spinal cord. J Neurophysiol 79:743-752.

Hultborn H, Jankowska E, Lindstrom S, Roberts W (1971) Neuronal pathway of the recurrent facilitation of motoneurones. J Physiol (Lond) 218:495-514.

Hultborn H, Lindstrom S, Wigstrom H (1979) On the function of recurrent inhibition in the spinal cord. Exp Brain Res 37:399-403.

Hwa GG, Avoli M (1991) Cesium potentiates epileptiform activities induced by bicuculline methiodide in rat neocortex maintained in vitro. Epilepsia 32:747-754.

Ichinose T, Miyata Y (1998) Recurrent excitation of motoneurons in the isolated spinal cord of newborn rats detected by whole-cell recording. Neurosci Res 31:179-187.

Jankowska E, Hammar I, Chojnicka B, Heden CH (2000) Effects of monoamines on interneurons in four spinal reflex pathways from group I and/or group II muscle afferents. Eur J Neurosci 12:701-714.

Jiang ZG, Shen E, Wang MY, Dun NJ (1991) Excitatory postsynaptic potentials evoked by ventral root stimulation in neonate rat motoneurons in vitro. J Neurophysiol 65:57-65.

Jonas P, Bischofberger J, Sandkuhler J (1998) Corelease of two fast neurotransmitters at a central synapse. Science 281:419-424.

Kiehn O, Kjaerulff O (1996) Spatiotemporal characteristics of 5-HT and dopamine-induced rhythmic hindlimb activity in the in vitro neonatal rat. J Neurophysiol 75:1472-1482.

Kiehn O, Kjaerulff O (1998) Distribution of central pattern generators for rhythmic motor outputs in the spinal cord of limbed vertebrates. Ann NY Acad Sci 860:110-129.

Kiehn O, Sillar KT, Kjaerulff O, McDearmid JR (1999) Effects of noradrenaline on locomotor rhythm-generating networks in the isolated neonatal rat spinal cord. J Neurophysiol 82:741-746.

Kraus T, Neuhuber WL, Raab M (2004) Vesicular glutamate transporter 1 immunoreactivity in motor endplates of striated esophageal but not skeletal muscles in the mouse. Neurosci Lett 360:53-56.

Machacek DW, Garraway SM, Shay BL, Hochman S (2001) Serotonin induces a long-lasting reflex facilitation in isolated rat spinal cord. J Physiol (Lond) 537:201-207.

Malomouzh AI, Mukhtarov MR, Nikolsky EE, Vyskocil F, Lieberman EM, Urazaev AK (2003) Glutamate regulation of non-quantal release of acetylcholine in the rat neuromuscular junction. J Neurochem 85:206-213.

Martin KA (2002) Microcircuits in visual cortex. Curr Opin Neurobiol 12:418-425.

McCrea DA, Pratt CA, Jordan LM (1980) Renshaw cell activity and recurrent effects on motoneurons during fictive locomotion. J Neurophysiol 44:475-488. 
McCurdy ML, Hamm TM (1994) Spatial and temporal features of recurrent facilitation among motoneurons innervating synergistic muscles of the cat. J Neurophysiol 72:227-234.

Mentis GZ, Alvarez FJ, Bonnot A, Richards DS, Gonzalez-Forero D, Zerda R, O’Donovan MJ (2005) Noncholinergic excitatory actions of motoneurons in the neonatal mammalian spinal cord. Proc Natl Acad Sci USA 102:7344-7349.

Moore RY, Card JP (1984) Noradrenaline-containing neuron systems. In: Handbook of chemical neuroanatomy (Bjorklund A, Hokfelt T, eds), pp 123-156. Amsterdam: Elsevier.

Nishimaru H, Restrepo CE, Ryge J, Yanagawa Y, Kiehn O (2005) Mammalian motor neurons corelease glutamate and acetylcholine at central synapses. Proc Natl Acad Sci USA 102:5245-5249.

Renshaw B (1941) Influence of discharge of motoneurons upon excitation of neighboring motoneurons. J Neurophysiol 4:167-183.
Schneider SP, Fyffe REW (1992) Involvement of GABA and glycine in recurrent inhibition of spinal motoneurons. J Neurophysiol 68:397-406.

Schwartzkroin PA, Scharfman HE, Sloviter RS (1990) Similarities in circuitry between Ammon's horn and dentate gyrus: local interactions and parallel processing. Prog Brain Res 83:269-286.

Shu Y, Hasenstaub A, McCormick DA (2003) Turning on and off recurrent balanced cortical activity. Nature 423:288-293.

Singer JH, Talley EM, Bayliss DA, Berger AJ (1998) Development of glycinergic synaptic transmission to rat brain stem motoneurons. J Neurophysiol 80:2608-2620.

Smith AD, Bolam JP (1990) The neural network of the basal ganglia as revealed by the study of synaptic connections of identified neurones. Trends Neurosci 13:259-265.

Wilson VJ, Burgess PR (1962) Disinhibition in the cat spinal cord. J Neurophysiol 25:392-404. 